\title{
Avaliação do desempenho de diferentes interfaces para ventilação não invasiva em modelo mecânico simulando paciente com DPOC
}

\section{Performance of different interfaces for noninvasive ventilation (NPPV) in a lung model simulating a COPD patient}

\author{
Francinni Mambrini Pires Rego', Letícia Zumpano Cardenas', Pedro Caruso², \\ Carlos Roberto Ribeiro Carvalho ${ }^{3}$, Juliana Carvalho Ferreira ${ }^{4}$
}

Rego FMP, Cardenas LZ, Caruso P, Carvalho CRR, Ferreira JC. Avaliação do desempenho de diferentes interfaces para ventilação nãoinvasiva em modelo mecânico simulando paciente com DPOC / Performance of different interfaces for noninvasive ventilation (NPPV) in a lung model simulating a COPD patient. Rev Med (São Paulo). 2012 abr.-jun.;91(2):60-8.

RESUMO: Ventilação mecânica não invasiva (VNI) é medida de suporte ventilatório comumente utilizada em pacientes com Doença Pulmonar Obstrutiva Crônica (DPOC). O objetivo deste estudo é comparar o desempenho de três interfaces para VNI em termos de sincronia paciente-ventilador. Utilizamos um simulador mecânico de pulmão alimentado e acoplado a uma cabeça de manequim para simular um paciente com DPOC em VNI. Três máscaras com volumes internos de 366, 550 e $1500 \mathrm{~mL}$ foram testadas. Os resultados mostraram que as máscaras com menor volume interno tiveram menor vazamento e consequentemente melhor sincronia com o simulador, com atraso de disparo mais curto (131 e 128ms vs 153ms) e aceleração de fluxo mais rápida (138 e 143ms vs $161 \mathrm{~ms})$. Concluímos que o tipo de interface usado para VNI tem impacto na sincronia e sua escolha pode ser guiada pela maior necessidade de aliviar o trabalho respiratório.

DESCRITORES: Ventilação não invasiva; Doença pulmonar obstrutiva crônica; Ventiladores mecânicos.

\begin{abstract}
Noninvasive ventilation (NPPV) is frequently used in Chronic Obstructive Pulmonary Disease (COPD). The aim of this study is to compare the performance of three NPPV interfaces in terms of patient-ventilator synchrony. We used a lung simulator attached to a mannequin head to simulate a COPD patient on NPPV. Three interfaces with inner volumes of 366,550 and $1500 \mathrm{~mL}$ were tested. Results showed that the interface with larger inner volume had higher air leakage, while smaller inner volume interfaces had better synchrony with the simulator, with shorter trigger delay (131 e $128 \mathrm{~ms}$ vs $153 \mathrm{~ms}$ ) and faster flow acceleration (138 e 143ms vs $161 \mathrm{~ms}$ ). We conclude that the type of interface used for NPPV has an impact on synchrony, and the choice of the interface may be based on the need to or unload respiratory muscles.
\end{abstract}

KEYWORDS: Noninvasive ventilation; Pulmonary disease, chronic obstructive; Ventilators, mechanical.

Prêmio Oswaldo Cruz - Áreca Clínica - COMU 2011.

1. Acadêmicas de Medicina da Faculdade de Medicina da Universidade de São Paulo.

2. Professor Colaborador da Disciplina de Pneumologia da Faculdade de Medicina da Universidade de São Paulo.

3. Professor Livre-docente da Faculdade de Medicina da Universidade de São Paulo.

4. Médica da UTI Respiratória do Hospital das Clínicas da Faculdade de Medicina da Universidade de São Paulo, LIM 09 - Laboratório de Investigação Médica - Pneumologia.

Endereço para correspondência: Divisão de Pneumologia do Instituto do Coração (InCor) da Faculdade de Medicina da USP. Av. Dr. Enéas de Carvalho Aguiar, 44, $5^{\circ}$ andar, sala 1. CEP: 05403-000, São Paulo, SP, E-mail: francinni.mambrini@gmail.com 
Rego FMP, et al. Avaliação do desempenho de diferentes interfaces para VNI.

\section{INTRODUÇÃO}

ventilação não invasiva (VNI) consiste no suporte ventilatório mecânico oferecido a paciente sem que seja necessária a colocação de via aérea artificial. Assim, o paciente não apresenta as complicações associadas à intubação endotraqueal, o risco de infecção associada à ventilação mecânica (VM) é reduzido e há preservação dos mecanismos de defesa das vias aéreas. Além disso, o conforto do paciente é maior, pois ele é capaz de mantera fala e deglutição e é mais facilmente transportado pelo hospital quando necessário. Finalmente, vale destacar a praticidade com que é realizada a retirada da interface de $\mathrm{VNI}$, possibitando desmame mais fácil da VM'. A Ventilação não invasiva mostrou-se uma estratégia efetiva para evitar a intubação e suas complicações em pacientes com Doença Pulmonar Obstrutiva Crônica (DPOC) e insuficiência respiratória, e para reduzir mortalidade nesses pacientes ${ }^{2-4}$. Seu uso em Unidades de Terapia Intensiva e mesmo em outros setores dos hospitais vem aumentando no mundo todo, embora diversos fatores justifiquem grandes variações regionais ${ }^{5-8}$, como a indisponibilidade de interfaces, o preço elevado em relação à cânula orotraqueal e a falta de profissionais habilitados ao seu uso. A VNI é atualmente considerada como tratamento de primeira linha para insuficiência respiratória aguda de diversas causas, entre elas edema pulmonar cardiogênico $0^{9-10}$, insuficiência respiratória em pacientes imunossuprimidos ${ }^{11,14}$, e especialmente, para pacientes com exacerbações agudas de DPOC $^{15}$. Os melhores resultados são observados em pacientes com insuficiência respiratória hipercápnica, pois a VNI aumenta a ventilação alveolar, ajudando a reduzir a $\mathrm{PaCO}_{2}$ e promovendo alívio da sobrecarga da musculatura respiratória.

Mesmo quando bem indicada, a VNI pode não ser efetiva, isto é, pode não evitar a intubação em até $40 \%$ dos casos $^{16}$. Uma das principais razões da falência da VNI é a intolerância do paciente à máscara, que é frequentemente ruidosa, pode levar a ferimentos na pele ao ser ajustada à face por longos períodos além da assincronia às vezes apresentada com o ventilador mecânico.

A interface utilizada para aplicar a VNI pode ser uma máscara nasal (cobre o nariz, mas não a boca), orofacial (cobre o nariz e a boca), facial total (cobre nariz, boca e olhos) ${ }^{17}$ ou um capacete (cobre toda a cabeça e parte do pescoço $)^{18}$.

As máscaras nasais são frequentemente usadas em pacientes com insuficiência respiratória crônica por cobrirem menor área do rosto, permitindo a fala e tosse, gerando maior tolerância ${ }^{19}$.
Entretanto, se o paciente mantiver a boca aberta e tiver respiração oral ou oro - nasal, sua eficiência fica limitada, e a monitorização do volume corrente ofertado deixa de ser confiável20.

As máscaras orofaciais são preferíveis em pacientes com insuficiência respiratória aguda, pois ao cobrir a boca garantem que o volume corrente expiratório seja monitorado e que não haja perda de pressurização no caso de haver respiração oronasal, não dependendo, desse modo, da colaboração do paciente. Sua principal desvantagem é frequentemente associar-se a lesões cutâneas e intolerância em longos períodos de uso ${ }^{20}$.

O capacete e as máscaras faciais totais surgiram como uma solução para a intolerância a máscaras orofaciais relacionadas a lesão de pele ou vazamentos na região próxima a base do nariz, que levam a ressecamento ocular. São mais confortáveis, pois não comprimem a base do nariz do paciente, que é um dos locais mais sujeitos a lesões quando se usa máscaras orofaciais, e distribuem melhor a pressão de fixação da máscara, gerando menos lesão cutânea ${ }^{20}$. Entretanto, seu volume interno e vazamento são maiores, o que pode comprometer a eliminação do $\mathrm{CO}_{2}$ e o alívio na sobrecarga da musculatura respiratória, reduzindo a eficácia da $\mathrm{VN}^{21-24}$. Em um estudo fisiológico, Vargas et al. ${ }^{25}$ demonstraram que o uso de pressões de suporte e PEEPs mais altas podem contrabalançar esse efeito nos capacetes. Seu uso, contudo, é heterodoxo e pode não ser aceito pelo paciente e família.

Diferentes interfaces e seu volume interno podem também influenciar o volume de vazamento e os padrões de pressão e fluxo dentro da máscara, e transmitidos ao paciente, influenciando assim a interação paciente-ventilador ${ }^{26-28}$. Interfaces com maior volume interno podem atenuar a transmissão de pressões entre ventilador e paciente, resultando em assincronia paciente-ventilador, o que leva à piora dos resultados da ventilação e é incômodo ao paciente.

Uma ressalva deve ser feita quando comparamos o volume interno de diferentes interfaces para VNI: o volume interno referido pelo fabricante pode ser bem diferente de volume interno medido depois que a interface está fixada no rosto do paciente.

Navalesi et al. ${ }^{19}$ compararam diferenças no volume interno entre uma máscara nasal e uma máscara oronasal, antes e depois de fixadas no rosto de um voluntário saudável. Apesar de a diferença antes da fixação ser grande, uma vez que a máscara oronasal era fixada ao rosto do paciente, seu volume interno caia bastante, e se assemelhava ao volume interno da máscara nasal também fixada ao rosto do 
voluntário. Este fenômeno pode ser importante para máscaras faciais totais, cujo volume interno é alto, mas que ao fixarem-se ao rosto do paciente, ficam com volume interno consideravelmente menor.

Além do volume interno da máscara medido in vitro, o padrão de fluxo dentro da máscara, a presença de vazamentos intencionais ou não intencionais ${ }^{29}$, variações de volume corrente e esforço do paciente, e uso de válvulas de exalação ${ }^{30,31}$ também influenciam o espaço morto efetivo da interface e a reinalação de $\mathrm{CO}_{2}$, podendo influenciar os resultados obtidos com a VNI. Um estudo recente em pacientes com insuficiência respiratória, mas estáveis o suficiente para tolerar turnos seqüenciais em quatro interfaces diferentes, mostrou que interfaces de alto volume podem ter desempenho comparável a interfaces de volume bem menor ${ }^{32}$.

Uma nova máscara facial total (Performax, Philips) foi desenvolvida para minimizar o volume interno e manter o ajuste confortável das máscaras faciais totais, não há dados na literatura sobre a sincronia paciente-ventilador associada a seu uso.

\section{OBJETIVOS}

Os objetivos de nosso estudo é:

1. avaliar o desempenho de três interfaces quanto à sincronia com os esforços inspiratórios durante a VNI em um modelo mecânico simulando um paciente com DPOC e insuficiência respiratória.

\section{MÉTODOS}

\section{A) Modelo mecânico}

O simulador ASL5000 (IngMar Medical; Pittsburgh, PA) é um modelo mecânico computadorizado que consiste em um pistão que se move dentro de um cilindro ${ }^{33}$. O simulador utiliza a equação do movimento, descrita abaixo, para controlar a posição do pistão dentro do cilindro a cada instante:

\section{Pva $=(E) \cdot(V)+(R) \cdot\left(V^{\prime}\right)+$ PEEP - Pmus}

Onde: $P v a=$ pressão de vias aéreas; $E$ = elastância, ou 1/complacência; $V$ = volume; $R=$ resistência; $V^{\prime}=$ fluxo; PEEP = pressão positiva ao final da expiração; Pmus = pressão muscular (esforço inspiratório).

Fluxo e pressão de vias aéreas são medidos por sensores de pressão e fluxo na entrada do pistão, e o volume é obtido por integração do fluxo no tempo. O simulador executa os cálculos necessários a 2000 $\mathrm{Hz}$ para controlar o movimento do pistão. Através de software específico, o usuário pode ajustar diversas características do sistema respiratório, entre elas a freqüência respiratória, complacência e resistência do sistema respiratório, e principalmente o perfil de esforço muscular (pressão negativa criada pelos músculos respiratórios) a cada ciclo respiratório ${ }^{34}$. Para este estudo, simulamos um paciente com Doença Pulmonar Obstrutiva Crônica (DPOC) com complacência de $80 \mathrm{~mL} / \mathrm{cm} \mathrm{H}_{2} \mathrm{O}$, resistência inspiratória de $10 \mathrm{~cm} \mathrm{H}_{2} \mathrm{O} / \mathrm{L} / \mathrm{s}$ e resistência expiratória de $20 \mathrm{~cm} \mathrm{H}$ O/L/s. O tempo inspiratório foi de 0,81 $\mathrm{s}$, e o pico de pressão muscular negativa (Pmus) foi ajustado para $5 \mathrm{~cm} \mathrm{H}_{2} \mathrm{O}$. A queda de pressão $100 \mathrm{~ms}$ após o início de um esforço ocluído (P0.1) foi $3.6 \mathrm{~cm}$ $\mathrm{H}_{2} \mathrm{O}$, e a freqüência respiratória $15 \mathrm{rpm}$. Para criar o perfil de esforço inspiratório (Pmus), utilizamos $5 \%$ do ciclo respiratório para esforço inspiratório inicial, 3\% de pausa no pico de esforço inspiratório e $15 \%$ do ciclo para retorno da Pmus para o repouso (Figura 1).

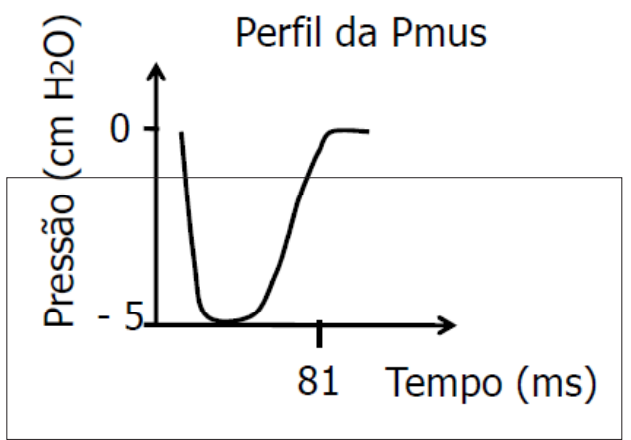

Figura 1. Perfil da Pmus simulando o esforço respiratório

\section{B) Montagem do experimento}

Uma cabeça de manequim adulto de fibra de vidro foi usada para simular a interface pacientemáscara. Tubos endotraqueais introduzidos na altura da boca e narinas direcionam o ar vindo da máscara para o simulador (Figura 2). Um ventilador mecânico (BiPap Vision, Philips) foi conectado à máscara acoplada à face do manequim por circuito próprio, de via única (Philips), com a válvula Whisper Swivel (Philips), conforme recomendação do fabricante para ventilação não invasiva com circuito único.

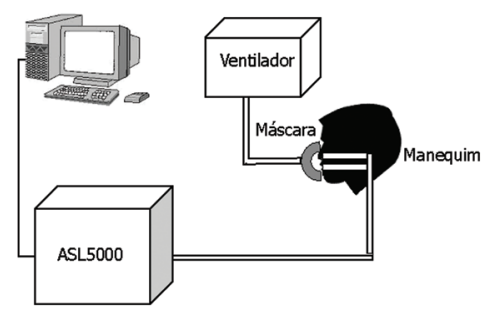

Figura 2. Montagem do experimento 
Rego FMP, et al. Avaliação do desempenho de diferentes interfaces para VNI.

\section{C) Interfaces testadas}

Três modelos de máscara foram fixadas à cabeça do manequim com faixas fixadoras, conforme recomendação dos fabricantes das máscaras. Testamos as seguintes interfaces:

1) máscara oral-nasal (Performatrak, Philips, tamanho G) (Orofacial);
2) máscara facial total (Total face, Philips, tamanho único) (Totalface);

3) máscara facial total (Performax, Philips, tamanho G) (Fullface). As interfaces foram firmemente ajustadas ao rosto do manequim para minimizar vazamentos de ar durante a VNI.

As características gerais das interfaces testadas encontram-se na Tabela 1.

Tabela 1. Características gerais das interfaces utilizadas

\begin{tabular}{ccccc}
\hline Tipo & Modelo & Fabricante & Tamanho & Volume interno \\
\hline Orofacial & Performatrack & Philips/Respironics & Grande & $336 \mathrm{~mL}$ \\
Totalface & Totalface & Philips/Respironics & Pequena & $1500 \mathrm{~mL}$ \\
Fullface & Performax & Philips/Respironics & Grande & $550 \mathrm{~mL}$ \\
\hline
\end{tabular}

\section{D) Protocolo experimental}

O ventilador foi ajustado para o modo espontâneo em dois níveis de pressão, com pressão inspiratória (IPAP) $=10 \mathrm{cmH}_{2} \mathrm{O}$, pressão expiratória $(E P A P)=5 \mathrm{cmH}_{2} \mathrm{O}$ e aceleração de fluxo inspiratório $=0.05 \mathrm{~s}$.

Um minuto de ventilação foi gravado para cada interface para análise das variáveis de interação paciente-ventilador descritas abaixo.

\section{E) Variáveis analisadas}

Para cada interface avaliada, foram registrados os valores de vazamento detectado pelo ventilador (Vaz), fornecidos pelo ventilador mecânico durante a aplicação da VNI. O ASL5000 fornece valores a cada respiração para diversas variáveis respiratórias, entre elas volume corrente (Vtidal); volume-minuto (VE), tempo de disparo (Ttrig), tempo para retorno à pressão de base (Tbase), pressão de disparo (Ptrig), atraso de ciclagem (Cdelay), calculado como a diferença entre o tempo inspiratório mecânico, registrado pelo $\mathrm{ASL} 5000^{34}$, e tempo inspiratório neural, programado para ser de $0.81 \mathrm{~s}$; e tempo para atingir $90 \%$ da pressão máxima (T90\%) (Figura 3).

Análise off-line de cada ciclo respiratório foi feita com software especifico do ASL5000 (Labview; National Instruments; Austin, TX).

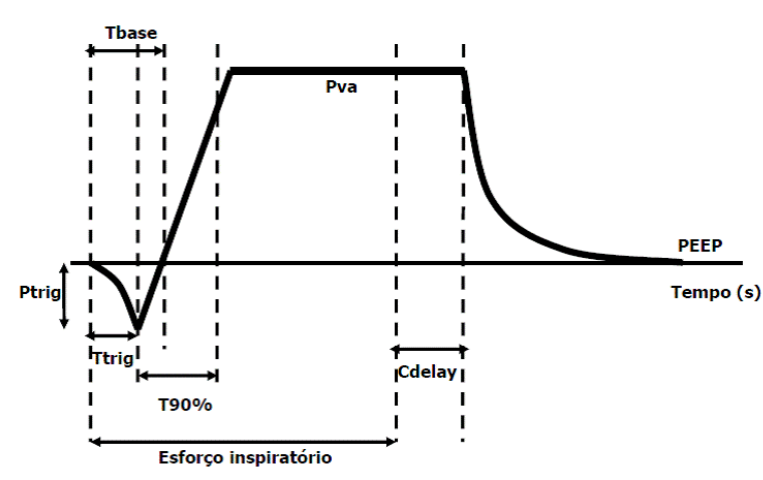

Figura 3. Variáveis obtidas com o ASL5000

\section{F) Análise estatística}

Valores estão expressos como média e desvio padrão. Usamos análise de variância (ANOVA) para medidas repetidas para comparar o desempenho das três interfaces. A comparação entre múltiplas médias foi realiazada pelo teste de Bonferoni (post-hoc). Consideramos valores de $\mathrm{p}<0,05$ como estatisticamente significantes.

\section{RESULTADOS}

A Tabela 2 mostra os resultados das variáveis de sincronia do experimento.

Tabela 2. Interação paciente-ventilador para cada interface ( ${ }^{*}$ indica $p<0,05$ à análise de ANOVA e ${ }^{\#}$ indica $p<0,05$ à comparação de Bonferroni)

\begin{tabular}{ccccccc}
\hline & Vtidal $(\mathrm{mL})$ & Tivent $(\mathrm{s})$ & Cdelay $(\mathrm{ms})^{\$}$ & ${\text { Ttrig }(\mathrm{ms})^{\$}}$ & Ptrig $\left(\mathrm{cmH}_{2} \mathrm{O}\right)$ & InspT90\% $(\mathrm{ms})^{\$}$ \\
\hline Orofacial & $241 \pm 1$ & $1,17 \pm 0,42$ & $366 \pm 42$ & $131 \pm 6$ & $-1,03 \pm 0,06$ & $137 \pm 6$ \\
Totalface & $240 \pm 8$ & $1,14 \pm 0,14$ & $332 \pm 142$ & $153 \pm 9^{\star}$ & $-0,99 \pm 0,13$ & $160 \pm 7^{\star}$ \\
Fullface & $242 \pm 3$ & $1,23 \pm 0,55^{\star}$ & $429 \pm 55^{\star}$ & $128 \pm 7$ & $-0,92 \pm 0,12^{*}$ & $142 \pm 5^{\#}$ \\
\hline
\end{tabular}

\$indica unidade $(m s)$ 
O tempo inspiratório do ventilador (Tivent) foi estatisticamente maior para a máscara Fullface em relação às duas outras interfaces $(p<0,001$ à análise de ANOVA). A diferença entre a máscara Totalface e a máscara Orofacial não foi estatisticamente significante $(p=0,0537$, à análise de Bonferroni) (Figura 4).

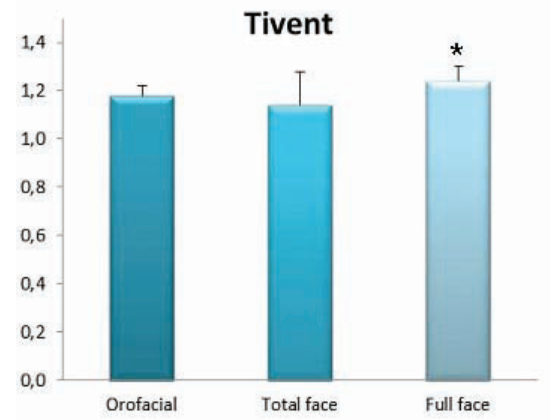

Figura 4. Tivent(s) de acordo com a interface ( ${ }^{*}$ indica $\left.p<0,05\right)$

Consequentemente, $\mathrm{o}$ atraso de ciclagem (Cdelay) foi maior para a máscara Fullface em relação à máscara orofacial $(p<0.001)$ e à Totalface $(p<0.001)$ (Tabela 2).

À semelhança do que ocorreu na análise da variável Tivent, o Cdelay não apresenta diferença estatisticamente significante quando se comparam as interfaces Totalface e Orofacial ( $p=0.537$ ) (Figura 5).

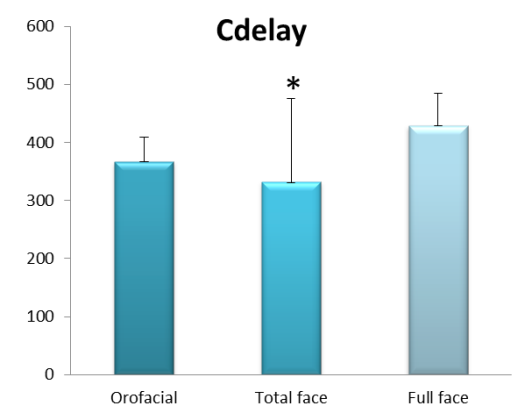

Figura 5. Atraso de ciclagem (Cdelay,ms) de acordo com a interface ${ }^{*}$ indica $\mathrm{p}<0,05$ )

$\mathrm{O}$ volume corrente (Vtidal) foi semelhante para as três interfaces $(p=0,396)$ (Figura 6).

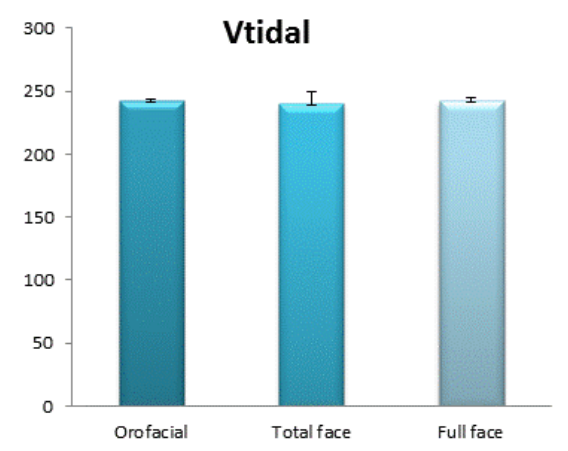

Figura 6. Volume corrente (Vtidal, $\mathrm{mL}$ ) de acordo com a interface
O tempo de disparo foi mais longo para a máscara Totalface em relação à máscara Orofacial e à máscara Fullface $(p<0.001)$. A comparação entre os valores obtidos com as interfaces Orofacial e Fullface não apresenta diferença signifativa $(p=0.520)$, conforme apresentado na Figura 7 .

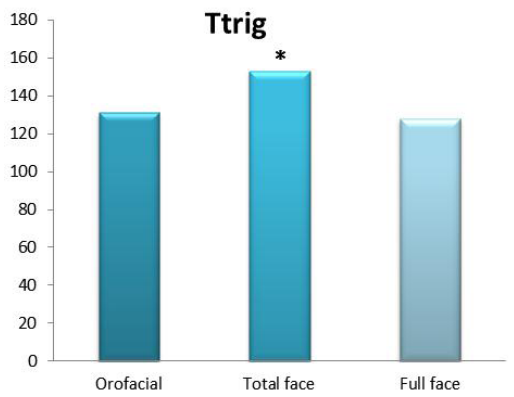

Figura 7. Tempo de disparo (Ttrig), em ms, de acordo com a interface, $\left({ }^{*}\right.$ indica $\left.p<0,05\right)$

A pressão de disparo (Ptrig), foi menor para a máscara Fullface em relação às máscaras Orofacial $(p=0,001)$ e Totalface $(p=0,001)$. Não houve diferenças entre as interfaces Orofacial e Totalface $(p=0,776)$, como ilustrado na Figura 8.

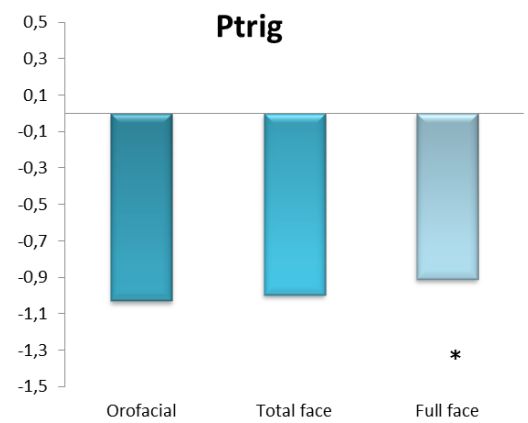

Figura 8. Pressão de disparo (Ptrig), em $\mathrm{cmH}_{2} \mathrm{O}$, para cada uma das interfaces $\left({ }^{*}\right.$ indica $\left.p<0,05\right)$

A análise do Tbase (ms) mostrou diferença significativa entre as três máscaras. $O$ Tbase mais longo foi encontrado para a máscara totalface, seguido pela Fullface e Orofacial $(p<0.001$ para todas as comparações), como mostrado na Figura 9.

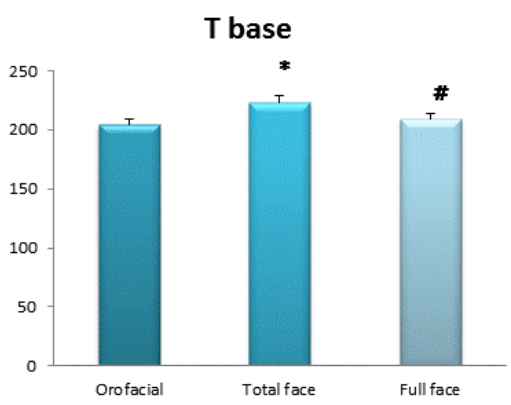

Figura 9. Valores de Tbase, em ms, para cada interface $\left({ }^{*}\right.$ indica $p<0,05$ à análise de ANOVA e \# indica $p<0,05$ à comparação de Bonferroni) 
Rego FMP, et al. Avaliação do desempenho de diferentes interfaces para VNI.

Finalmente, o tempo para atingir $90 \%$ da pressão máxima (T90\%) foi mais longo $(p=0,009)$ com a Totalface do que para as máscaras Fullface e Orofacial ( $p<0.001$ para ambas as comparações), e foi também mais longo para a máscara Fullface do que para a máscara Orofacial $(p=0.009)$, como mostra a Figura 10.

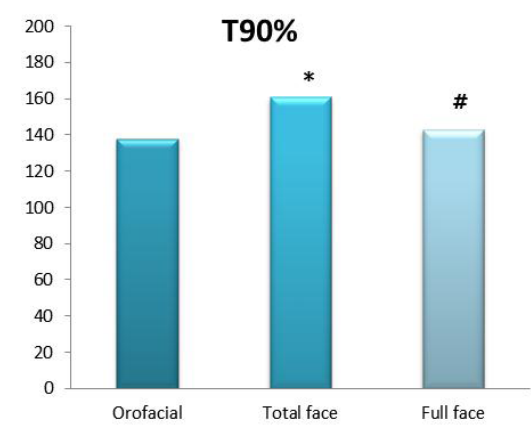

Figura 10. Tempo em ms para atingir $90 \%$ da pressão máxima (T90\%) de acordo com a interface. ( ${ }^{*}$ indica $p<0,05$ à análise de ANOVA $e$ \#indica $p<0,05$ à comparação de Bonferroni

\section{DISCUSSÃO}

O uso da técnica de ventilação não-invasiva é cada vez mais comum em enfermarias, unidades de terapia intensiva e pronto-socorros em especial para pacientes com Doença Pulmonar Obstrutiva Crônica (DPOC) em exacerbação e na vigência de hipercapnia, situação na qual seu uso já está muito bem estabelecido ${ }^{35}$. Os bons resultados refletiram-se em interesse sobre o assunto na literatura e levaram à realização de estudos clínicos de boa qualidade, muitos dos quais realizados na última década. Como consequência, a VNI apresenta grau A de recomendação ${ }^{35}$ para tratar insuficiência respiratória hipercápnica, sendo as principais indicações a presença de dispnéia moderada a grave, $\mathrm{pH}<7,35$ com $\mathrm{pCO}_{2}>45$ e frequência respiratória maior que 25ipm 1 .

Os bons resultados levaram também ao crescente interesse da indústria em produzir acessórios para realização da $\mathrm{VNI}^{36}$. Graças ao interesse comercial, diversos tipos de máscaras foram criados, com diferentes indicações, tamanhos, mecanismos e preços. A dúvida em relação a qual interface ser utilizada surgiu e novos experimentos foram delineados a fim de que as vantagens e desvantagens de cada modelo fossem dissecadas. Como as diferenças entre as máscaras são, não raramente, discretas, existe a demanda de grande exatidão no delineamento destes trabalhos. Novas interfaces são lançadas no mercado sem que existam estudos suficientes que comprovem seus reais benefícios em relação às antigas. A classe médica é exposta a esses novos produtos e, à semelhança do que ocorre em outras especialidades, sem que qualquer informação de melhor qualidade seja transmitida, ficando a cargo do usuário o teste do produto.

Dados subjetivos avaliando o conforto relacionado ao diferentes modelos foram publicados $^{28,30}$, mas o estudo da sincronia pacienteventilador em uso de diferentes interfaces ainda é limitado. Quando existem, esses estudos, por questões éticas, são realizados em pacientes saudáveis, que testam múltiplas interfaces na vigência da avaliação pretendida. O estudo do comportamento das interfaces quando aplicadas em condições críticas, por exemplo, através destes estudos é muito difícil.

Somam-se à grande variedade de interfaces e situações clínicas, as dificuldades técnicas para medição dos padrões ventilatórios in vivo ${ }^{36}$. Tratase de parâmetros muito sensíveis, cuja análise não suporta variações mínimas de condições ambientais. É o caso, por exemplo, do vazamento. Durante nossos experimentos, notamos grande variação do vazamento encontrado quando uma mesma máscara era fixada de diferentes modos por pessoas diferentes ao nosso modelo.

Nuances como estas dificultam a realização de ensaios clínicos sobre o tema que apresentem boa qualidade. Contudo, a qualidade dos estudos experimentais também é limitada. A complexidade da dinâmica ventilatória impossibilita a criação de um modelo ideal. Os simuladores de pulmão de funcionamento puramente mecânico, como o TTL da Michigan Instruments (Dual Adult TTL Michigan Instruments, Michigan, USA), muito usado em estudos de bancada, não têm capacidade de reproduzir diversas propriedades relacionadas à dinâmica respiratória e permitem apenas ajustes de resistência e complacência. Além disso, seu funcionamento depende de um ventilador extra, que não será avaliado e que é responsável por manter a dinâmica do sistema durante os experimentos. Em nosso experimento utilizamos um modelo relativamente novo, computadorizado, baseado no fluxo de ar gerado a partir do movimento de um pistão dentro de um cilindro de ar. O movimento do pistão é realizado a partir da equação do movimento do sistema respiratório, conforme descrevemos na seção Métodos. A determinação dos valores de fluxo ins e expiratório, pressão de vias aéreas e volume corrente é produto de uma análise extremamente complexa em que diversas variáveis relacionadas à mecânica ventilatória são consideradas. O software do modelo exige amplo conhecimento da fisiologia respiratória e permite ajustar não apenas o formato da curva da pressão 
muscular durante o ciclo respiratório, mas também uma série de outras variáveis, desde a possibilidade de variação da complacência, resistência, tempos respiratórios, volumes, características do fluxo aéreo, características das vias aéreas, ventilação pulmonar ou alveolar, pressão em vários pontos da via aérea, entre outros.

Com base na determinação de todos estes parâmetros, a equação do movimento é aplicada e controla o movimento do pistão a cada instante do ciclo respiratório, com uma freqüência de $2000 \mathrm{~Hz}$. $\mathrm{O}$ resultado da interação entre o pistão e a VNI aplicada ao sistema gera as curvas que podem ser analisadas no software, de acordo com o interesse do pesquisador.

Para este estudo, escolhemos avaliar a sincronia com o simulador de três usando um modelo de paciente com DPOC.

A análise do tempo inspiratório do ventilador (Tivent) e do tempo de atraso de ciclagem (Cdelay), que é dele derivado, demonstrou que o maior valor absoluto foi obtido ao testarmos a máscara Fullface. A análise dos valores de volume corrente (Vtidal) não apresentou diferença entre as três máscaras. O tempo de disparo foi maior quando avaliados os dados referentes à máscara Totalface e de forma análoga, o valor do módulo da pressão de disparo, Ptrig, foi menor para a Fullface. A análise de Tbase mostrou diferença entre todas as máscaras, sendo o valor menor relacionado à máscara Orofacial e o mais longo relacionado à máscara Totalface. Finalmente, o tempo para atingir $90 \%$ da pressão máxima foi superior quando utilizada a máscara Totalface e mínimo para a máscara Orofacial.

Os resultados encontrados estão condizentes com achados descritos em outros trabalhos.

A máscara Orofacial tem características físicas, em especial o menor volume interno, que minimizam o vazamento, colaborando para a sincronia pacienteventilador. Os resultados demonstraram que ela atingiu esta meta em relação aos outros modelos analisados. Embora a diminuição da superfície de contato da máscara com o rosto do paciente leve à redução do vazamento, protegendo a qualidade da sincronia e reduzindo o esforço respiratório e prevenindo a fadiga muscular, ela também se traduz em elevação da pressão a que é submetida a pele, levando a feridas. Como consequência, seu uso é limitado, por exemplo, em pacientes idosos que apresentam pele naturalmente friável. $O$ tempo de uso da máscara também pode ser reduzido em consequência às feridas. Finalmente, vale destacar que este modelo pode ser incômodo para pacientes com nível de consciência mais preservado, uma vez que o vazamento de grandes quantidades de ar na região do nariz leva a importante ressecamento ocular.

A máscara Totalface revelou-se inferior às outras interfaces analisadas quando se aborda a sincronia ventilador-paciente. Este modelo de máscara foi desenvolvido para priorizar o conforto para pacientes com intolerância às máscaras orofaciais, e em quem se antecipa duração prolongada do uso da VNI. Por cobrir todo o rosto, evita a compressão da base do nariz e vazamentos de ar para a região dos olhos. Por outro lado, tem um volume interno muito maior, e vazamentos intencionais para minimizar a reinalação de $\mathrm{CO}_{2}$ consequente a esse volume mais alto, o que aumenta o vazamento total e limita seu uso a ventiladores que conseguem compensar tais vazamentos. Além disso, o vazamento maior pode interferir na transmissão de pressões dentro da máscara, o que por sua vez tem impacto na sincronia entre paciente e ventilador. As máscaras faciais totais foram criadas a fim de que as lesões de pele causadas pelas máscaras de VNI no rosto dos pacientes fossem minimizados. $\mathrm{O}$ aumento do volume interno dessas máscaras poderia dificultar a lavagem do $\mathrm{CO}_{2}$, que se acumularia no sistema e contribuiria para piorar as condições clínicas em pacientes graves. A solução do problema foi a criação de dois orifícios frontais no modelo, por onde grandes quantidades de ar pressurizado escapam, auxiliando de forma na lavagem do $\mathrm{CO}_{2}$. Os vazamentos de grande monta percebidos nos nossos resultados são explicados em parte por este fato. Um segundo item a ser observado é o grande perímetro da interface, que, em contato com as irregularidades da pele da face proporcionam diversos pontos de vazamento não controláveis.

A máscara Fullface foi criada na tentativa de somar as qualidades dos dois outros modelos. Conforme corroborado pelos resultados, o seu desempenho é bom quando analisada a sincronia paciente-ventilador. Sua superfície de contato com a pele é intermediária quando comparada aos dois outros modelos. Ela é grande o suficiente para impedir as lesões nasais geradas pela máscara Orofacial uma vez que se apóia na fronte do paciente, bem como o ressecamento ocular relacionado ao vazamento pelas bordas da máscara, representando opção confortável, com boa aceitação pelos pacientes e familiares. $O$ modelo não apresenta orifícios frontais, sendo o uso da válvula whisper swivel também indicado a fim de otimizar a lavagem de $\mathrm{CO}_{2}$ que é considerada intermediária. Trata-se, portanto de uma opção com bons resultados quando aplicada a pacientes que necessitam de diminuição do resforço respiratório e que apresentam níveis de capnia elevados ou mesmo para quem se antecipe duração prolongada do uso de VNI. 


\section{CONCLUSÃO}

A partir dos resultados pudemos notar que assim como na prática clínica foram observados vazamentos de grande quantidade secundários à adaptação imperfeita das máscaras ao rosto do modelo.

Devido aos grandes vazamentos observados, encontramos um aumento de aproximadamente $50 \%$ no tempo inspiratório do ventilador em relação ao tempo de esforço inspiratório realizado pelo modelo mecânico de pulmão, o que é consistente com outros estudos de bancada encontrados na literatura.

\section{REFERÊNCIAS}

1. Oliveira AR, Taniguchi LU, Park M, et al. Manual de residência de medicina intensiva.2a ed. rev. ampl. São Paulo: Manole; 2011.

2. Brochard L, Mancebo J, Wysocki M, Lofaso F, Conti G, Rauss A, Simonneau G, Benito S, Gasparetto $A$, Lemaire F: Noninvasive ventilation for acute exacerbations of chronic obstructive pulmonary disease. N Engl J Med. 1995;333:817-;22.

3. Ambrosino N, Vagheggini G. Noninvasive positive pressure ventilation in the acute care setting: where are we? Eur Respir J. 2008;31:874-86.

4. III Consenso Brasileiro de Ventilação Mecânica. J Bras Pneumol. 2007;33(Supl 2):S 92-S105.

5. Demoule A, Girou E, Richard JC, Taillé S, Brochard L. Increased use of noninvasive ventilation in French intensive care units. Intensive Care Med. 2006;32:1747-55.

6. Esteban A, Ferguson ND, Meade MO, et al., Evolution of mechanical ventilation in response to clinical research, Am J Respir Crit Care Med. 2008;177:1707.

7. Crimi C, Noto A, Esquinas A, Nava S. Non-invasive ventilation (NIV) practices: a European web-survey. Eur Respir J. 2008;32:1970.

8. Maheshwari V, Paioli D, Rothaar R, Hill N. Utilization of noninvasive ventilation in acute care hospitals: a regional survey, Chest. 2006;129:1226-33.

9. Park M, Lorenzi-Filho G. Noninvasive mechanical ventilation in the treatment of acute cardiogenic pulmonary edema. Clinics. 2006;61:247-52.

10. Vital FM, Saconato H, Ladeira MT, Sen A, Hawkes CA, Soares B, Burns KE, Atallah AN. Non-invasive positive pressure ventilation (CPAP or bilevel NPPV) for cardiogenic pulmonary edema. Cochrane Database Syst Rev. 2008;16(3):CD005351.

11. Antonelli M, Conti G, Bufi $M$, et al. Noninvasive ventilation for treatment of acute respiratory failure in patients undergoing solid organ transplantation: a randomized trial. JAMA. 2000;283:235-41.

12. Depuydt PO, Benoit DD, Vandewoude $\mathrm{KH}$, Decruyenaere JM, Colardyn FA. Outcome in
As máscaras Orofacial e Fullface apresentaram bom desempenho quando avaliamos parâmetros relacionados à sincronia. Em relação a estas variáveis, a máscara Totalface não apresentou desempenho tão satisfatório.

Desta forma, conclui-se que as máscaras Orofacial e Fullface são mais bem indicadas quando a diminuição do esforço respiratório e alívio do trabalho da musculatura respiratória do paciente é primordial.

Estudos clínicos, em pacientes com insuficiência respiratória, avaliando o desempenho dessas interfaces são necessários para confirmar nossos achados.

noninvasively and invasively ventilated hematologic patients with acute respiratory failure. Chest. 2004;126:1299-306.

13. Hilbert G, Gruson D, Vargas F, et al. Noninvasive ventilation in immunosuppressed patients with pulmonary infiltrates, fever, and acute respiratory failure. N Engl J Med. 2001;344:481-7.

14. Confalonieri M, Calderini E, Terraciano $S$, et al. Noninvasive ventilation for treating acute respiratory failure in AIDS patients with Pneumocystis carinii pneumonia. Intensive Care Med. 2002;28:1233-8.

15. Ram FS, Picot J, Lightowler J, Wedzicha JA. Noninvasive positive pressure ventilation for treatment of respiratory failure due to exacerbations of chronic obstructive pulmonary disease. Cochrane Database Syst Rev. 2004(3):CD004104.

16. Carlucci A, Richard JC, Wysocki M, et al: Noninvasive versus conventional mechanical ventilation. An epidemiologic survey. Am J Respir Crit Care Med. 2001;163:874-80.

17. Criner GJ, Travaline JM, Brennan KJ, Kreimer DT. Efficacy of a new full face mask for non-invasive positive pressure ventilation. Chest. 1994;106:110915.

18. Antonelli M, Conti G, Pelosi P, Gregoretti C, Pennini MA, Costa R, et al. New treatment of acute hypoxemic respiratory failure: noninvasive pressure support ventilation delivered by helmet- a pilot controlled trial. Crit Care Med. 2002;30:602-8.

19. Navalesi $P$, Fanfulla F, Frigerio P, Gregoretti C, Nava S. Physiologic evaluation of noninvasive mechanical ventilation delivered with three types of masks in patients with chronic hypercapnic respiratory failure. Crit Care Med. 2000,28:1785-90.

20. Nava S, Navalesi P, Gregoretti C. Interfaces and humidification for noninvasive mechanical ventilation. Respir Care. 2009;54:71-84.

21. Gonzalez J, Sharshar T, Hart N, Chadda K, Raphael JC, Lofaso F. Air leaks during mechanical ventilation as a cause of persistent hypercapnia in neuromuscular disorders. Intensive Care Med. 2003;29:596-602. 
22. Taccone P, Hess D, Caironi P, Bigatello LM. Continuous positive airway pressure delivered with a "helmet": effects on carbon dioxide rebreathing. Crit Care Med. 2004;32:2090-6.

23. Patroniti N, Foti G, Manfio A, Coppo A, Bellani $G$, Pesenti A. Head helmet versus face mask for non-invasive continuous positive airway pressure: a physiological study. Intensive Care Med. 2003:29:1680-7.

24. Antonelli M, Pennisi MA, Pelosi P, Gregoretti C, Squadrone V, Rocco M, Cecchini L, Chiumello $D$, Severgnini $P$, Proietti R, Navalesi P, Conti G. Noninvasive positive pressure ventilation using a helmet in patients with acute exacerbation of chronic obstructive pulmonary disease: a feasibility study. Anesthesiology. 2004;100:16-24.

25. Vargas F, Thille A, Lyazidi A, Campo FR, Brochard L. Helmet with specific settings versus facemask for noninvasive ventilation. Crit Care Med. 2009;37:19218.

26. Schettino GP, Tucci MR, Sousa R, Barbas V, Amato MBP, Carvalho CRR. Mask mechanics and leak dynamics during noninvasive pressure support ventilation: a bench study. Intensive Care Med. 2001;27:1887-91.

27. Mehta S, McCool FD, Hill NS. Leak compensation in positive pressure ventilators: a lung model study. Eur Respir J. 2001;17:259-67.

28. Navalesi $P$, Costa $R$, Ceriana $P$, Carlucci $A$, Prinianakis $G$, et al. Non-invasive ventilation in chronic obstructive pulmonary disease patients: helmet versus facial mask. Intensive Care Med. 2007;33:74-81.

29. Fodil R, Lellouche F, Mancebo J, Sbirlea-Apiou
G, Isabey D, Brochard L, Louis B. Comparison of patient-ventilator interfaces based on their computerized effective dead space. Intensive Care Med. 2011;37:257-62.

30. Schettino GP, Chatmongkolchart S, Hess DR, Kacmarek MR. Position of exhalation port and mask design affect $\mathrm{CO}_{2}$ rebreathing during noninvasive positive pressure ventilation. Crit Care Med. 2003;31:2178-82.

31. Ferguson GT, Gilmartin $\mathrm{M}$. $\mathrm{CO}_{2}$ rebreathing during BiPAP ventilatory assistance. Am J Respir Crit Care Med. 1995;151(4):1126-35.

32. Fraticelli AT, Lellouche F, L'her E, Taillé S, Mancebo $\mathrm{J}$, Brochard L. Physiological effects of different interfaces during noninvasive ventilation for acute respiratory failure. Crit Care Med. 2009;37:939-45.

33. IngMar Medical. ASL5000 Active Servo Lung Computerized Breathing Simulator and Ventilator Test Instrument user's manual. Pittsburgh, PA: IngMar Medical; 2006.

34. Ferreira J, Chipman DW, Kacmarek RM. Trigger performance of mid-level ICU mechanical ventilators during assisted ventilation: a bench study. Intensive Care Med. 2008;34:1669-75.

35. Schettino GPP, coordenador. III Consenso Brasileiro de Ventilação Mecânica. Cap. 3: Ventilação mecânica não-invasiva com pressão positiva. J Bras Pneumol. 2007;33(Supl. 2):S92-S105. Disponível em: htt://www.jornaldepneumologia.com.br/PDF/ Suple_151_47_3cap3.pdf

36. Keenan SP, Wenston B. Interfaces para ventilação não-invasiva: faz diferença? [Editorial]. J Bras Pneumol. 2009;35(2):103-5. 